\title{
Certain class of higher-dimensional simplicial complexes and universal $C^{*}$-algebras
}

\author{
Saleh Omran ${ }^{1,2}$
}

\begin{abstract}
In this article we introduce a universal $C^{*}$-algebras associated to certain simplicial flag complexes. We denote it by $\mathcal{C}_{\Gamma^{n}}$ it is a subalgebra of the noncommutative $n$-sphere which introduced by J.Cuntz. We present a technical lemma to determine the quotient of the skeleton filtration of a general universal $C^{*}$-algebra associated to a simplicial flag complex. We examine the $K$-theory of this algebra. Moreover we prove that any such algebra divided by the ideal $/ 2$ is commutative.
\end{abstract}

2000 AMS: 19 K 46

Keywords: Simplicial complexes; K- theory of $C^{*}$-algebras; Universal $C^{*}$-algebras

\section{Introduction}

In this section, we give a survey of some basic definitions and properties of the universal $C^{*}$-algebra associated to a certain flag complex which we will use in the sequel. Such algebras in general was introduced first by Cuntz (2002) and studied by Omran $(2005,2013)$.

Definition 1. A simplicial complex $\Sigma$ consists of a set of vertices $V_{\Sigma}$ and a set of non-empty subsets of $V_{\Sigma}$, the simplexes in $\Sigma$, such that:

- If $s \in V_{\Sigma}$, then $\{s\} \in \Sigma$.

- If $F \in \Sigma$ and $\emptyset \neq E \subset F$ then $E \in \Sigma$.

A simplicial complex $\Sigma$ is called flag or full, if it is determined by its 1-simplexes in the sense that $\left\{s_{0}, \ldots, s_{n}\right\} \in$ $\Sigma \Longleftrightarrow\left\{s_{i}, s_{j}\right\} \in \Sigma$ for all $0 \leq i<j \leq n$.

$\Sigma$ is called locally finite if every vertex of $\Sigma$ is contained in only finitely many simplexes of $\Sigma$, and finitedimensional (of dimension $\leqslant n$ ) if it contains no simplexes with more than $n+1$-vertices. For a simplicial complex $\Sigma$ one can define the topological space $|\Sigma|$ associated to this complex. It is called the "geometric realization" of the complex and can be defined as the space of maps

\footnotetext{
Correspondence: salehomran@yahoo.com

1 Faculty of Science, Taif University, Taif, Kingdom of Saudi Arabia

2 Faculty of science, Math. Department, KSA South valley university, Qena, Egypt
}

\section{照 Springer}

C 2014 Omran; licensee Springer. This is an Open Access article distributed under the terms of the Creative Commons Attribution License (http://creativecommons.org/licenses/by/2.0), which permits unrestricted use, distribution, and reproduction in any medium, provided the original work is properly credited. $f: V_{\Sigma} \longrightarrow[0,1]$ such that $\sum_{s \in V_{\Sigma}} f(s)=1$ and $f\left(s_{0}\right) \ldots .$. $f\left(s_{i}\right)=0$ whenever $\left\{s_{0}, \ldots, s_{i}\right\} \notin \Sigma$. If $\Sigma$ is locally finite, then $|\Sigma|$ is locally compact.

Let $\Sigma$ be a locally finite flag simplicial complex. Denote by $V_{\Sigma}$ the set of its vertices. Define $\mathcal{C}_{\Sigma}$ as the universal $C^{*}$ algebra with positive generators $h_{s}, s \in V$, satisfying the relations

$$
\begin{aligned}
& h_{s_{0}} h_{s_{1}} \ldots h_{s_{n}}=0 \text { whenever }\left\{s_{0}, s_{1}, \ldots, s_{n}\right\} \notin V_{\Sigma}, \\
& \sum_{s \in V_{\Sigma}} h_{s} h_{t}=h_{t} \quad \forall t \in V_{\Sigma} .
\end{aligned}
$$

Here the sum is finite, because $\Sigma$ is locally finite.

$\mathcal{C}_{\Sigma}^{a b}$ is the abelian version of the universal $C^{*}$-algebra above, i.e. satisfying in addition $h_{s} h_{t}=h_{t} h_{s}$ forall $s, t \in$ $V_{\Sigma}$. Denote by $I_{k}$ the ideal in $\mathcal{C}_{\Sigma}$ generated by products containing at least $n+1$ different generators. The filtration $\left(I_{k}\right)$ of $\mathcal{C}_{\Sigma}$ is called the skeleton filtration.

Let

$$
\Delta:=\left\{\left(s_{0}, \ldots, s_{n}\right) \in \mathbb{R}^{n+1} \mid 0 \leq s_{i} \leq 1, \sum_{i=1}^{n} s_{i}=1\right\}
$$

be the standard $n$-simplex. Denote by $\mathcal{C}_{\Delta}$ the associated universal $C^{*}$-algebra with generators $h_{s}, s \in\left\{s_{0}, \ldots, s_{n}\right\}$, such that $h_{s} \geq 0$ and $\sum_{s} h_{s}=1$. Denote by $\mathcal{I}_{\Delta}$ the ideal in $\mathcal{C}_{\Delta}$ generated by products of generators containing all the $h_{s_{i}}, i=0, \ldots, n$. For each $k$, denote by $I_{k}$ the ideal in $\mathcal{C}_{\Delta}$ generated by all products of generators $h_{s}$ containing at least $k+1$ pairwise different generators. We also denote by $I_{k}^{a b}$ the image of $I_{k}$ in $\mathcal{C}_{\Delta}^{a b}$. The algebra $\mathcal{C}_{\Delta}$ and their 
$K$-Theory was studied in details in (Omran and Gouda 2012). For any vertex $t$ in $\Delta$ there is a natural evaluation map $\mathcal{C}_{\Delta} \longrightarrow \mathbb{C}$ mapping the generators $h_{t}$ to 1 and all the other generators to 0 . The following propositions are due to Cuntz (2002).

Proposition 1. (i) The evaluation map $\mathcal{C}_{\Delta} \longrightarrow \mathbb{C}$ defined above induces an isomorphism in $K$-theory. (ii) The surjective map $\mathcal{I}_{\Delta} \longrightarrow \mathcal{I}_{\Delta}^{a b}$ induces an isomorphism in $K$-theory, where $\mathcal{I}_{\Delta}^{a b}$ is the abelianization of $\mathcal{I}_{\Delta}$.

We can observe that $I_{k}$ is the kernel of the evaluation map which define above so we can conclude that $I_{k}$ is closed.

Remark 1. Let $\Delta$ and $\mathcal{I}_{\Delta} \subset \mathcal{C}_{\Delta}$ as above. Then $K_{*}\left(\mathcal{I}_{\Delta}\right) \cong K_{*}(\mathbb{C}), *=0,1$, if the dimension $n$ of $\triangle$ is even and $K_{*}\left(\mathcal{I}_{\Delta}\right) \cong K_{*}\left(C_{0}(0,1)\right), *=0,1$, if the dimension $n$ of $\triangle i$ s odd.

Proposition 2. Let $\Sigma$ be a locally finite simplicial complex. Then $\mathcal{C}_{\Sigma}^{a b}$ is isomorphic to $C_{0}(|\Sigma|)$, the algebra of continuous functions vanishing at infinity on the geometric realization $|\Sigma|$ of $\Sigma$.

\section{Universal $C^{*}$-algebras associated to certain complexes}

Universal $C^{*}$-algebras is a $C^{*}$-algebras generated by generators and relations. Many $C^{*}$-algebras can be constructed in the form of universal $C^{*}$-algebras an important example for universal $C^{*}$-algebras is Cuntz algebras $O_{n}$ the existence of this algebras and their $K$-theory was introduced by Cuntz $(1981,1984)$ more other examples of universal $C^{*}$-algebras can be found in (Cuntz 1993; Davidson 1996). In the following, we introduce a general technical lemma to compute the quotient of the skeleton filtration for a general algebra associated to simplicial complex.

For a subset $W \subset V_{\Sigma}$, let $\Gamma \subset \Sigma$ be the subcomplex generated by $W$ and let $\mathcal{I}_{\Gamma}$ be the ideal in $\mathcal{C}_{\Gamma}$ generated by products containing all generators of $\mathcal{C}_{\Gamma}$.

Lemma 1. Let $\mathcal{C}_{\Sigma}$ and $\mathcal{C}_{\Gamma}$ as above, then we have

$$
I_{k} / I_{k+1} \cong \bigoplus_{W \subset V_{\Sigma},|W|=k+1} \mathcal{I}_{\Gamma}
$$

Proof. $\mathcal{C}_{\Sigma} / I_{k+1}$ is generated by the images $\dot{h}_{i}, i \in V_{\Sigma}$ of the generators in the quotient.

Given a subset $W \subset V_{\Sigma}$ with $|W|=k+1$, let

$$
\mathcal{C}_{\Gamma^{\prime}}=C^{*}\left(\left\{\dot{h_{i}} \mid i \in W\right\}\right) \subset \mathcal{C}_{\Sigma} / I_{k+1} .
$$

Let $\mathcal{I}_{\Gamma^{\prime}}$ denote the ideal in $\mathcal{C}_{\Gamma^{\prime}}$ generated by products containing all generators $\dot{h}_{i}, i \in \Gamma^{\prime}$, and let $\mathcal{B}_{\Gamma}$ denote its closure. If $W \neq W^{\prime}$, then $\mathcal{B}_{\Gamma} \mathcal{B}_{\Gamma^{\prime}}=0$, because the product of any two elements in $\mathcal{B}_{\Gamma}$ and $\mathcal{B}_{\Gamma^{\prime}}$ contains products of more than $k+1$-different generators, which are equal to zero in the algebra $\mathcal{C}_{\Sigma} / I_{k+1}$

It is clear that $\mathcal{B}_{\Gamma} \subset I_{k} / I_{k+1}$ so that

$$
\bigoplus_{W \subset V_{\Sigma},|W|=k+1} \mathcal{B}_{\Gamma} \subset I_{k} / I_{k+1} .
$$

Conversely, let $x \in I_{k} / I_{k+1}$. Then there is a sequence $\left(x_{n}\right)$ converging to $x$, such that each $x_{n}$ is a sum of monomials $m_{s}$ in $\dot{h}_{i}$ containing at least $k+1$-different generators. Then $m_{s} \in \mathcal{B}_{\Gamma}$ for some $W$ and

$$
x_{n}=\sum m_{s} \in \bigoplus_{W \subset V_{\Sigma},|W|=k+1} \mathcal{B}_{\Gamma} .
$$

The space $\bigoplus_{W \subset V,|W|=k+1} \mathcal{B}_{\Gamma}$ is closed, because it is a direct sum of closed ideals. It follows that

$$
I_{k} / I_{k+1}=\bigoplus_{W \subset V_{\Sigma},|W|=k+1} \mathcal{B}_{\Gamma}
$$

Let now

$$
\pi_{W}: \mathcal{C}_{\Sigma} \longrightarrow \mathcal{C}_{\Gamma}
$$

be the canonical evaluation map defined by

$$
\pi_{W}\left(h_{i}\right)= \begin{cases}h_{i}^{\prime} & \forall i \in W \\ 0 & \text { if } i \notin W,\end{cases}
$$

where $h_{i}{ }^{\prime}$ denotes the generator in $\mathcal{C}_{\Gamma}$ corresponding to the index $i$ in $W$, in other words

$$
\mathcal{C}_{\Gamma}=C^{*}\left(h_{i}^{\prime} \mid i \in W\right) .
$$

We prove that $\pi_{W}\left(I_{k+1}\right)=0$. Since polynomials of the form

$\sum \ldots h_{i_{0}} \ldots h_{i_{j}} \ldots h_{i_{k+1}} \ldots, \quad i_{0}, \ldots, i_{j}, \ldots, i_{k+1}, \ldots \in V_{\Sigma}$

are dense in $I_{k+1}$, it is enough to show that $\pi_{W}(x)=0$ for each such polynomial $x$. We have

$$
\pi_{W}(x)=\sum \ldots h_{i_{0}}^{\prime} \ldots h_{i_{j}}^{\prime} \ldots h_{i_{k+1}}^{\prime} \ldots=0,
$$

since there is at least one $i_{l}$ which is not in $W$. For this index $\pi_{W}\left(h_{i_{l}}\right)=0$. Thus $\pi_{W}(x)=0$. Therefore $\pi_{W}$ descends to a homomorphism

$$
\pi_{W}: \mathcal{C}_{\Sigma} / I_{k+1} \longrightarrow \mathcal{C}_{\Gamma}
$$

Now we show that $\pi_{W}$ is surjective as follows: Since $\pi_{W}\left(I_{k+1}\right)=0$, we have $\operatorname{Ker} \pi_{W} \supset I_{k+1}$. It follows that the following diagram

$$
\begin{array}{ccc}
\mathcal{C}_{\Sigma} \longrightarrow & \begin{array}{c}
\mathcal{C}_{\Gamma} \\
\searrow \\
\end{array} \\
& \mathcal{C}_{\Sigma} / I_{k+1}
\end{array}
$$

commutes and $\pi_{W}\left(\dot{h}_{i}\right):=\pi_{W}\left(h_{i}\right)=h_{i}{ }^{\prime}, i \in W$ is well defined. This shows that $\pi_{W}\left(\mathcal{C}_{\Sigma}\right)$ is a closed subalgebra in 
$\mathcal{C}_{\Gamma}$ and isomorphic to $\pi_{W}\left(\mathcal{C}_{\Sigma} / I_{k+1}\right)$. We have $\pi_{W}\left(\mathcal{B}_{\Gamma}\right)=$ $\mathcal{I}_{\Gamma}$. It is clear that Ker $\pi_{W}$ is the ideal generated by $h_{i}$ for $i$ not in $W$ and therefore Ker $\pi_{W}$ is generated by $\dot{h}_{i}$ for $i$ not in $W$. This comes at once from the definitions of $\pi_{W}\left(\dot{h}_{i}\right)$ and $\pi_{W}\left(h_{i}\right)$ above and the fact that both are equal. We conclude that $\mathcal{B}_{\Gamma} \operatorname{Ker} \pi_{W}=0$. This again implies that $\mathcal{B}_{\Gamma} \cap \operatorname{Ker} \pi_{W}=0$. Moreover the following diagram is commutative:

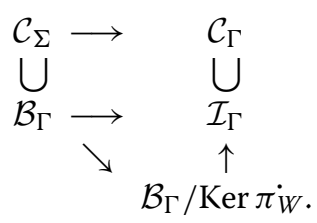

So, $\pi_{W}\left(\mathcal{B}_{\Gamma}\right)$ is dense and closed in $\mathcal{I}_{\Gamma}$. Therefore $\pi_{W}$ : $\mathcal{B}_{\Gamma} \longrightarrow \mathcal{I}_{\Gamma}$ is injective and surjective.

As a consequence of the above lemma we have the following.

Proposition 3. Let $\mathcal{C}_{\Delta}$ and $I_{k}$ defined as above. Then we have an isomorphism

$$
I_{k} / I_{k+1} \cong \bigoplus_{\Delta} \mathcal{I}_{\triangle}
$$

where the sum is taken over all k-simplexes $\triangle$ in $\Sigma$.

Proof. As in the proof of lemma 1 above with $\Sigma=\Delta$, we find that:

$$
I_{k} / I_{k+1}=\bigoplus_{\Delta} \mathcal{I}_{\Delta}
$$

In the following we study the $C^{*}$-algebras $\mathcal{C}_{\Gamma^{n}}$ associated to simplicial flag complexes $\Gamma$ of a specific simple type. These simplicial complexes is a subcomplex of the "noncommutative spheres" in the sense of Cuntz work (Cuntz 2002). We determine the $K$-theory of $\mathcal{C}_{\Gamma^{n}}$ and also the $K$-theory of its skeleton filtration. The $K$-theory of $C^{*}$ algebras is a powerful tool for classifying $C^{*}$-algebras up to their Projections and unitaries, more details about $K$ theory of $C^{*}$-algebras found in the references (Blackadar 1986; Murphy 1990; Rørdam et al. 2000; Wegge-Olsen 1993).

We denote by $\Gamma^{n}$ the simplicial complex with $n+2$ vertices, given in the form

$$
V_{\Gamma^{n}}=\left\{0^{+}, 0^{-}, 1, \ldots, n\right\},
$$

and

$$
\Gamma^{n}=\left\{\gamma \subset V_{\Gamma^{n}} \mid\left\{0^{+}, 0^{-}\right\} \nsubseteq \gamma\right\} .
$$

Let

$$
\begin{aligned}
\mathcal{C}_{\Gamma^{n}} & =C^{*}\left(h_{0^{-}}, h_{0^{+}}, h_{1}, h_{2}, \ldots, h_{n} \mid h_{0^{-}} h_{0^{+}}\right. \\
& \left.=0, h_{i} \geqslant 0, \sum_{i} h_{i}=1, \forall i\right)
\end{aligned}
$$

be the universal $C^{*}$ - algebra associated to $\Gamma^{n}$. The existence of such algebras is due to Cuntz (2002). It is clear that for any element $h_{i} \in \mathcal{C}_{\Gamma^{n}}$, we have $\left\|h_{i}\right\| \leq 1$.

Denote by $\mathcal{I}$ the natural ideal in $\mathcal{C}_{\Gamma^{n}}$ generated by products of generators containing all $h_{i}, i \in V_{\Gamma^{n}}$. Then we have the skeleton filtration

$$
\mathcal{C}_{\Gamma^{n}}=I_{0} \supset I_{1} \supset I_{2} \supset \ldots . . \supset I_{n+1}:=\mathcal{I}
$$

The aim of this section is to prove that the $K$-theory of the ideals $\mathcal{I}$ in the algebras $\mathcal{C}_{\Gamma^{n}}$ is equal to zero. We have the following

Lemma 2. Let $\mathcal{C}_{\Gamma^{n}}$ be as above. Then $\mathcal{C}_{\Gamma^{n}}$ is homotopy equivalent to $\mathbb{C}$.

Proof. Let $\beta: \mathbb{C} \longrightarrow \mathcal{C}_{\Gamma^{n}}$ be the natural homomorphism which sends 1 to $1_{\mathcal{C}_{\Gamma^{n}}}$. For a fixed $i \in V_{\Gamma^{n}}$ such that $i \neq$ $0^{-}, 0^{+}$, define the homomorphism

$$
\alpha: \mathcal{C}_{\Gamma^{n}} \longrightarrow \mathbb{C}
$$

by $\alpha\left(h_{i}\right)=1$ and $\alpha\left(h_{j}\right)=0$ for any $j \neq i$. Notice that $\alpha \circ \beta=i d_{\mathbb{C}}$. Now define $\varphi_{t}: \mathcal{C}_{\Gamma^{n}} \longrightarrow \mathcal{C}_{\Gamma^{n}}, h_{i} \longmapsto h_{i}+$ $(1-t)\left(\sum_{j \neq i} h_{j}\right), h_{j} \longmapsto t\left(h_{j}\right) j \in V_{\Gamma^{n}} \backslash\{i\}$. The elements $\varphi_{t}\left(h_{j}\right), j \in V_{\Gamma^{n}}$, satisfy the same relations as the elements $h_{j}$ in $\mathcal{C}_{\Gamma^{n}}$ :

(i) $\varphi_{t}\left(h_{j}\right) \geq 0$

(ii) $\varphi_{t}\left(\sum_{j} h_{j}\right)=\varphi_{t}\left(h_{i}\right)+\sum_{j \neq i} \varphi_{t}\left(h_{j}\right)$

$$
\begin{aligned}
& =h_{i}+(1-t)\left(\sum_{j \neq i} h_{j}\right)+t\left(\sum_{j \neq i} h_{j}\right) \\
& =h_{i}+\sum_{j \neq i} h_{j} \text { for fixed } i \\
& =\sum_{j} h_{j}=1 \text { for all } j,
\end{aligned}
$$

(iii) $\varphi_{t}\left(h_{0^{-}}\right) \varphi_{t}\left(h_{0^{+}}\right)=t^{2}\left(h_{0^{-}} h_{0^{+}}\right)=0$.

We note that $\varphi_{1}=i d_{\mathcal{C}_{\Gamma^{n}}}$ and $\varphi_{0}=\beta \circ \alpha$.

This implies that

$$
\varphi_{0}=\beta \circ \alpha \sim I d_{\mathcal{C}_{\Gamma^{n}}} .
$$

This means that $\mathcal{C}_{\Gamma^{n}}$ is homotopy equivalent to $\mathbb{C}$.

From the above lemma, we have $K_{*}\left(\mathcal{C}_{\Gamma^{n}}\right)=K_{*}(\mathbb{C})$, for $*=0,1$. 
Now we describe the subquotients of the skeleton filtration in $\mathcal{C}_{\Gamma^{n}}$.

Proposition 4. In the $C^{*}$-algebra $\mathcal{C}_{\Gamma^{n}}$ one has

$$
I_{k} / I_{k+1} \cong \bigoplus_{\triangle} \mathcal{I}_{\triangle} \oplus \bigoplus_{\gamma} \mathcal{I}_{\gamma}
$$

where the sum is taken over all subcomplexes $\triangle$ of $\Gamma^{n}$ which are isomorphic to the standard $k$-simplex $\triangle$ and over all subcomplexes $\gamma$ of $\Gamma^{n}$ which contain both vertices $0^{+}, 0^{-}$ and the second sum is taken over every subcomplex $\gamma$ which contains both vertices $0^{+}, 0^{-}$and whose number of vertices is $k+1$.

Proof. We use Lemma 1 above. For every $W \subset V_{\Gamma^{n}}$ with $|W|=k+1$, we have two cases. Either $\left\{0^{+}, 0^{-}\right\}$is not a subset of $W$, then $\Gamma$ is a $k$-simplex, or $\left\{0^{+}, 0^{-}\right\}$is a subset of $W$, then $\Gamma$ is a subcomplex in $\Gamma^{n}$ isomorphic to $\gamma$. This proves our proposition.

Lemma 3. For the complex $\Gamma^{n}$ with $n+2$ vertices, $\mathcal{C}_{\Gamma^{n}} / I_{1}$ is commutative and isomorphic to $\mathbb{C}^{n+2}$.

Proof. Let $\dot{h}_{i}$ denote the image of a generator $h_{i}$ for $\mathcal{C}_{\Gamma^{n}}$. One has the following relations:

$$
\sum_{i} \dot{h}_{i}=1, \quad \dot{h}_{i} \dot{h}_{j}=0, \quad i \neq j .
$$

For every $\dot{h}_{i}$ in $\mathcal{C}_{\Gamma^{n}} / I_{1}$ we have

$$
\dot{h}_{i}=\dot{h}_{i}\left(\sum_{i} \dot{h_{i}}\right)=\dot{h}_{i}^{2} \text {. }
$$

Hence $\mathcal{C}_{\Gamma^{n}} / I_{1}$ is generated by $n+2$ different orthogonal projections and therefore $\mathcal{C}_{\Gamma^{n}} / I_{1} \cong \mathbb{C}^{n+2}$.

Lemma 4. $I_{1} / I_{2}$ in $\mathcal{C}_{\Gamma^{n}}$ is isomorphic to $I_{1}^{a b} / I_{2}^{a b}$ in $\mathcal{C}_{\Gamma^{n}}^{a b}$.

Proof. From the proposition 4 above, one has

$$
I_{1} / I_{2} \cong \bigoplus_{\triangle^{1}} \mathcal{I}_{\triangle^{1}}
$$

where $\Delta^{1}$ is 1 -simplex, and

$$
I_{1}^{a b} / I_{2}^{a b} \cong \bigoplus_{\triangle^{1}} \mathcal{I}_{\Delta^{1}}^{a b}
$$

Since $\mathcal{I}_{\triangle^{1}} \subset \mathcal{C}_{\triangle^{1}}$ is commutative because the generators of $\mathcal{C}_{\triangle^{1}}$ commute (since $h_{s_{1}}=1-h_{s_{0}}$ ). We get

$$
\mathcal{I}_{\triangle^{1}} \cong \mathcal{I}_{\Delta^{1}}^{a b} \cong C_{0}(0,1)
$$

Lemma 5. In $\mathcal{C}_{\Gamma^{n}}$, we have $K_{0}\left(I_{1} / I_{2}\right)=0$ and $K_{1}\left(I_{1} / I_{2}\right)=\mathbb{Z}^{\left(\begin{array}{c}n \\ 2\end{array}\right)+2 n}$.
Proof. By applying above lemma , and proposition 4, we have

$$
I_{1} / I_{2} \cong \bigoplus_{\triangle^{1}} \mathcal{I}_{\triangle^{1}}
$$

The sum contain $\left(\begin{array}{l}n \\ 2\end{array}\right)+2 n$ 1-simplex, $\Delta^{1} \cong C_{0}(0,1)$. where $K_{0}\left(C_{0}(0,1)\right)=0$ and $K_{1}\left(C_{0}(0,1)\right)=\mathbb{Z}$.

Lemma 6. $\mathcal{C}_{\Gamma^{n}} / I_{2}$ is a commutative $C^{*}$-algebra.

Proof. Consider the extension

$$
0 \longrightarrow I_{1} / I_{2} \longrightarrow \mathcal{C}_{\Gamma^{n}} / I_{2} \longrightarrow \mathcal{C}_{\Gamma^{n}} / I_{1} \longrightarrow 0
$$

and the analogous extension for the abelianized algebras.

The extensions above induce the following commutative diagram:

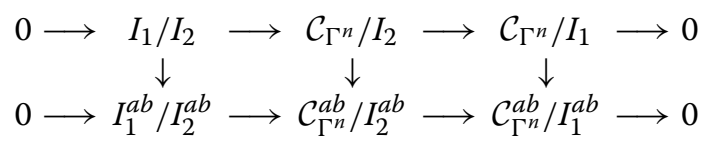

We have from 3 isomorphisms $\mathcal{C}_{\Gamma^{n}} / I_{1} \cong \mathcal{C}_{\Gamma^{n}}^{a b} / I_{1}^{a b} \cong \mathbb{C}^{n+2}$ and from 4 that $I_{1} / I_{2} \cong I_{1}^{a b} / I_{2}^{a b}$, so

$$
\mathcal{C}_{\Gamma^{n} / I_{2}} \cong \mathcal{C}_{\Gamma^{n}}^{a b} / I_{2}^{a b}
$$

Lemma 7. $C^{*}$-algebra $\mathcal{C}_{\Gamma^{1}}$ is commutative and $K_{*}\left(I_{2}\right)=$ $0, *=0,1$ where $I_{2}$ is an ideal in $\mathcal{C}_{\Gamma^{1}}$ defined as in the above.

Proof. $\mathcal{C}_{\Gamma^{1}}$ is generated by three positive generators, $h_{0^{-}}, h_{0^{+}}, h_{1}$. Consider the product of two generators, say $h_{1} h_{0^{-}}$. We have that $1, h_{0^{-}}$and $h_{0^{+}}$commute with $h_{0^{-}}$, therefore also $h_{1}=1-h_{0^{-}}-h_{0^{+}}$.

By a similar computation we can show that $h_{0^{+}}$and $h_{1}$ commute. This implies that $\mathcal{C}_{\Gamma^{1}}$ is commutative. Therefore $I_{2}=0$ in $\mathcal{C}_{\Sigma^{1}}$ Then, at once $K_{*}\left(I_{2}\right)=0$.

\section{Competing interests}

The author declare that he has no competing interests.

Received: 20 December 2013 Accepted: 17 March 2014 Published: 21 May 2014

\section{References}

Blackadar B (1986) K-theory for operator algebras. MSRI Publ. 5, Cambridge University Press

Cuntz J (1981) K-theory for certain $C^{*}$-algebras. Ann Math 113:181-197 Cuntz J (1984) K-theory and $C^{*}$-algebras. Proc. Conf. on K-theory (Bielefeld,1982). Springer Lecture Notes in Math. 1046:55-79 Cuntz J (1993) A survey of some aspects of noncommutative geometry. Jahresber D Dt Math-Verein 95:60-84

Cuntz J (2002) Non-commutative simplicial complexes and Baum-Connes-conjecture. GAFA, Geom Func Anal 12: 307-329 Davidson KR (1996) C*-algebras by example. Fields Institute monographs, Amer. Math. Soc, Providence

Murphy GJ (1990) C*-algebras and operator theory. Academic Press Omran S (2005) $C^{*}$-algebras associated with higher-dimensional noncommutative simplicial complexes and their K-theory. Dissertation, Münster: Univ. Münster-Germany 
Omran S (2013) $C^{*}$-algebras associated noncommutative circle and their K-theory. Aust J Math Anal Appl 10(1): 1-8. Article 8

Omran S, Gouda GhY (2012) On the K-theory of C*-algebras associated with n-Simplexes. Int J Math Anal 6(17): 847-855

Rørdam M, Larsen F, Laustsen N (2000) An introduction to K-theory for $C^{*}$-algebras. London Mathematical Society Student Text 49, Cambridge University Press

Wegge-Olsen NE (1993) K-theory and $C^{*}$-algebras. Oxford University Press, New York

\section{doi:10.1186/2193-1801-3-258}

Cite this article as: Omran: Certain class of higher-dimensional simplicial complexes and universal $C^{*}$-algebras. SpringerPlus 2014 3:258.

\section{Submit your manuscript to a SpringerOpen ${ }^{\circ}$} journal and benefit from:

- Convenient online submission

- Rigorous peer review

- Immediate publication on acceptance

- Open access: articles freely available online

- High visibility within the field

- Retaining the copyright to your article

Submit your next manuscript at $\gg$ springeropen.com 